\title{
Fabrication of Electrospun Membranes based on Poly(caprolactone) (PCL) and PCL/Chitosan Layer by Layer for Tissue Engineering
}

\author{
Choi Yee Foong ${ }^{1} \&$ Naznin Sultana ${ }^{1,2^{*}}$ \\ ${ }^{1}$ Faculty of Biosciences and Medical Engineering, \\ ${ }^{2}$ Advanced Membrane Technology Research Centre (AMTEC), \\ Universiti Teknologi Malaysia, 81310 UTM Johor Bahru, Johor, Malaysia
}

\begin{abstract}
Recently, in the field of tissue engineering, fabrication of three-dimensional (3D) electrospun scaffold or membrane is much emphasized. In this study, layered composite scaffolds or membranes were fabricated using two biodegradable polymers, polycaprolactone (PCL) and Chitosan layer-by-layer with multilayer electrospinning method. Characterizations of membranes were done using several techniques. Electrospun composite membrane's surface morphology was examined using a Scanning Electron Microscopy (SEM) and the wettability of the material's surface was determined using water contact angle measuring measurement (WCA). Water uptake properties of electrospun membrane were also determined. Using optimized solution concentration and electrospinning processing parameters, the composite PCL/Chitosan and PCL layer-by-layer were successfully fabricated. It was observed from SEM that the composite electrospun membranes produced consisted microfibers and nanofibers within single scaffold. The water contact angle for the double-layered composite electrospun membranes was lower than the pure PCL. The double-layered composite membrane also had higher water uptake properties compared to pure PCL scaffold.
\end{abstract}

Keywords: 3D Scaffold, Multilayer Electrospinning Technique, PCL, PCL/Chitosan, Tissue Engineering, SEM, water contact angle

\subsection{INTRODUCTION}

Recently, development of three dimensional (3D) scaffolds becomes one of the most challenging projects in the field of tissue engineering [1-5]. The production of 3D scaffold with different size and geometry is introduced. In terms of size, the 3D scaffolds can be in the combination of microscale and nanoscale while in terms of geometry, the 3D scaffolds produced consist of fibers and filaments $[6,7]$. The 3D scaffold network intervention is more suitable to be used in tissue engineering due to its highly interconnected structure as well as the increased surface area to volume ratio. This will enhance and promote the cell proliferation and migration $[8,9]$.

PCL is non-toxic, biocompatible and biodegradable polymer while its degradation rate is depending on the molecular weight of polyester. For higher molecular weight of PCL, the degradation time is about two to three years and it is not suitable for the short term implantations $[10,11]$. PCL has a few constraints such as it is hydrophobic, limited bio regulatory activity and neutral charge distribution [12]. On the other hand, Chitosan is a natural polysaccharides whose physical properties are identical to glycosaminoglycan [13]. In addition, Chitosan is non-toxic and biodegradable and thus it is safe to be applied in human body [14].Besides that, it has a good permeability for the oxygen and carbon dioxide to pass through it $[15,16]$. However, natural scaffold having poor control of enzymatic degradation and poor 
mechanical performance limits it's application in the tissue engineering [17]. By blending PCL and chitosan, the high mechanical strength of PCL and the hydrophilicity of the chitosan can be obtained in the same composite scaffold.

Electrospinning method is a promising technique for the fabrication of scaffold. The advantage of electrospinning method is that it is able to fabricate polymer scaffold fibers' size in a wide range [18]. The morphologies of fibers are affected by the nature of electrospinning and also the translation of polymer solution into nanofibers through electrospinning. There are several parameters such as solution parameters, processing parameter, and ambient parameters that can affect the final morphology of the membranes. We can make electrospun fibers with desired morphologies and diameters by proper control of those parameters [19].

When severe skin burn occurred, the ordinary pathway to solve the problem is using tissue engineering for tissue replacement and regeneration. However, conventional monolayer scaffold just consist of either nanofibers or microfibers and thus unable to get the advantages of both nanofibers and microfibers in single scaffold. Considering this problem, creation of suitable multi-layered scaffold for tissue engineering application is required. This paper reports the fabrication and characterization of layer by layer composite membrane based on polycaprolactone (PCL) and PCL/Chitosan through electrospinning techniques to be used in tissue regeneration.

\subsection{EXPERIMENTAL}

\subsection{Materials}

PCL with the molecular weight about $70,000-90,000$ was purchased from Aldrich (Germany). Chitosan as powder form was also purchased from Aldrich. All the solvents such as Chloroform, Formic Acid and Acetic Acid were analytical grade which were purchased from Sigma.

\subsection{Dope Preparation}

In order to prepare pure $13 \% \mathrm{w} / \mathrm{v}$ PCL polymer solution, $1.30 \mathrm{~g}$ of PCL was dissolved in $10 \mathrm{ml}$ of Chloroform by magnetically stirring at 500rpm and at $50^{\circ} \mathrm{C}$. On the other hand, in order to produce $8.8 \% \quad \mathrm{w} / \mathrm{v} \quad \mathrm{PCL} /$ Chitosan polymer solution, $0.8 \mathrm{~g}$ of $\mathrm{PCL}$ and $0.08 \mathrm{~g}$ of Chitosan were dissolved in $7 \mathrm{ml}$ of Formic Acid and $3 \mathrm{ml}$ of Acetic Acid by magnetically stirring at 500rpm at room temperature.

\subsection{Electrospinning}

Pure PCL, PCL/Chitosan, as well as PCL/Chitosan and PCL layer-by-layer membranes were fabricated by electrospinning method (NaBond Nanofiber Electrospinning Unit, China). Firstly, pure PCL (1.30g) polymer solution was inserted to a new $6 \mathrm{~cm} / \mathrm{ml}$ syringe with needle. After that, the syringe was placed in a syringe pump. An aluminum foil with surface area of $10 \mathrm{~cm} X 10 \mathrm{~cm}$ was prepared before the electrospinning started and being used as a collector. The processing parameters for each scaffold were different. Table 1 shows the summary of the parameters used for each electrospun membranes fabrication.

For the fabrication of PCL/Chitosan and PCL layer-by-layer electrospun 
membrane, all the steps involved in the electrospinning process were repeated and the parameters were unchanged. The time taken for the electrospinning process (pure PCL) was half an hour. After that, the same collector was used so that the PCL/Chitosan nanofibers were collected on top of the PCL fibers. The time taken for electrospinning process (PCL/Chitosan) was half an hour as well.

Table 1 Parameters used for the Fabrication of Pure PCL and PCL/Chitosan through Electrospinning Process

\begin{tabular}{ccc}
\hline Parameters & PCL & 'CL/Chitosan \\
\hline Tip-to-collector & 0.00 & 12.50 \\
Distance $(\mathrm{cm})$ & & \\
Temperature $\left({ }^{\circ} \mathrm{C}\right)$ & $\vdots 2.50$ & 22.50 \\
Humidity $(\%)$ & 17.00 & 51.00 \\
'low rate $(\mathrm{ml} / \mathrm{min})$ & 0.01 & 0.02 \\
Ioltage Used $(\mathrm{kV})$ & 7.40 & 20.00 \\
\hline
\end{tabular}

\subsection{Characterization}

The morphology of pure PCL, PCL/Chitosan as well as PCL/Chitosan and PCL layer-by-layer polymer fiber were observed using a Scanning Electron Microscope (Hitachi 400). A contact angle measuring system (VCA Optima, AST Products, Inc.) was used to determine the wettabilities of PCL, $\mathrm{PCL} /$ Chitosan as well as PCL/Chitosan and PCL layer-by-layer.

Electrospun membranes collected on the aluminum foil were peeled out and then double-tape was used to fix the polymer fiber on a coin. Each sample fixed on a coin. Titanium coating on the polymer fiber will provide a better result of SEM. An Image $J$ software was used to measure the diameter and pore sizes of fibers. At least 25 fiber diameters and pores were measured and the average was calculated.

In order to measure water contact angle of the membrane surfaces, the electrospun membranes were placed on a sample stage. The water drop was controlled by the measuring system. The sample table was turned upwards and downwards in order to allow water dropped from the machine onto the surface and check the wettability of samples. The process was repeated for at least five times.

Water uptake by the polymer fiber was conducted to check its hydrophilicity. The initial dry weight was recorded prior to the experiment. Next, the electrospun membranes were immersed in distilled water for $2,5,10$, $15,20,25,30,45$, and 60 minutes. Then, the electrospun membranes' weight was recorded for each time period by removing them from distilled water periodically. Electrospun membranes were dried using a filter paper in order to eliminate excess water. The equation below was used to calculate the water uptake by the microfibers membranes:

Wateruptake $(\%)=\left(W_{w}-W_{d}\right) / W_{d} \times 100$

where $\mathrm{W}_{\mathrm{d}}$ is dry weight and $\mathrm{W}_{\mathrm{w}}$ is wet weight of samples that obtained periodically.

\subsection{RESULTS AND DISCUSSION}

Figure 1 shows the morphology of the membranes. It was observed that PCL/Chitosan and PCL layer-by-layer were successfully fabricated and it consists of microfibers and nanofibers in one scaffold structure. Figure 2 shows the distribution of electrospun fiber diameter of $8.8 \% \quad \mathrm{w} / \mathrm{v}$ $\mathrm{PCL} /$ Chitosan and $13.0 \% \mathrm{w} / \mathrm{v}$ PCL micro/nano fibrous membrane layerby-layer. $13.0 \% \mathrm{w} / \mathrm{v}$ PCL displayed the largest average pore size which was about $1230.00 \mathrm{~nm}$ or $1.23 \mu \mathrm{m}$. On the other hand, the average fiber diameter 
Table 2 The diameter range of fibers in different membranes

\begin{tabular}{lcl}
\hline \multicolumn{1}{c}{ Membranes } & $\begin{array}{c}\text { Diameter Range } \\
(\mathbf{n m})\end{array}$ & $\begin{array}{c}\text { Average } \\
\text { Diameter } \\
(\mathbf{n m})\end{array}$ \\
\hline Pure PCL & $1002.00-2196.00$ & 1230.00 \\
PCL /Chitosan & $72.30-105.00$ & 93.54 \\
PCL and PCL /Chitosan Layer-by-Layer & $74.30-1281.00$ & 856.32 \\
\hline
\end{tabular}
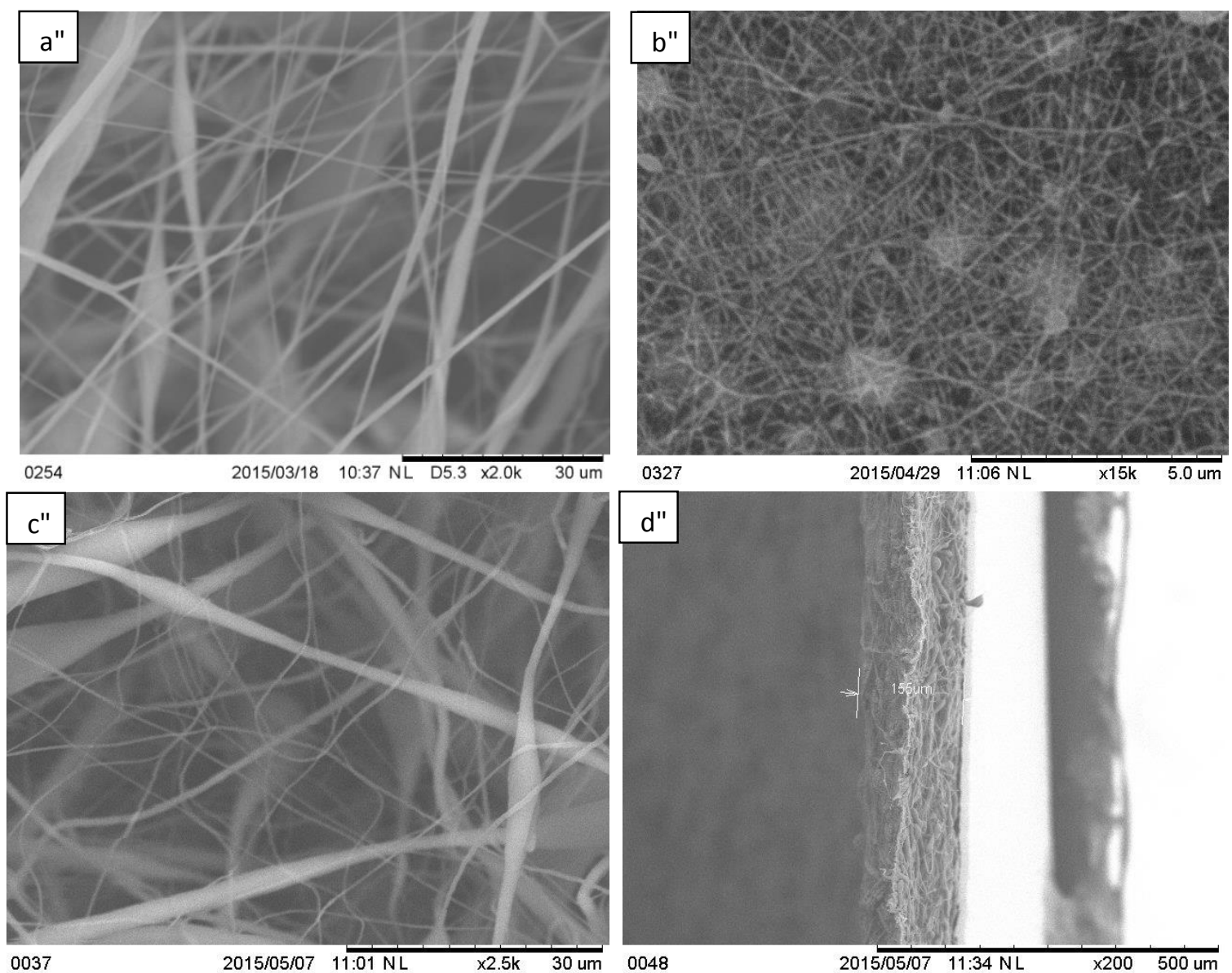

Figure 1 Scanning electron micrographs of electrospun membranes; (a) 13.0\% PCL using 7.40kV with magnification 2000, (b) $8.8 \%$ PCL/Chitosan using 20kVwith magnification 15000 , (c) $8.8 \%$ PCL/Chitosan and 13.0\% PCL layer-by-layer with magnification 2500(d) Cross Section of $8.8 \%$ PCL/Chitosan and 13,0\% PCL layer-by-layer with magnification 200

for the $8.8 \% \mathrm{w} / \mathrm{v}$ was the smallest which was $93.54 \mathrm{~nm}$ while the $13.0 \% \mathrm{w} / \mathrm{v}$ PCL and $8.88 \% \quad \mathrm{w} / \mathrm{v} \quad \mathrm{PCL} /$ Chitosan micro/nano fibrous membrane showed the intermediate average fiber diameter which was $856.32 \mathrm{~nm}$. Table 2 shows the mean value of fiber diameter for PCL, PCL/Chitosan as well as double layered PCL and PCL/Chitosan. The $13 \% \quad \mathrm{w} / \mathrm{v} \quad \mathrm{PCL}$ and $8.8 \mathrm{w} / \mathrm{v}$ PCL/Chitosan layer by layer membrane's fiber diameter were ranged 
from $74.3 \mathrm{~nm}$ to $1281 \mathrm{~nm}$. In other words, the layer by layer scaffold had the microfibers and nanofibers in single scaffold.

Fabrication of multilayer scaffold which consists of microfibers and nanofibers in order to get the advantages of both scales of fiber diameters in single scaffold is promising in tissue engineering [20]. The conventional monolayer electrospun membrane possesses microfibers or nanofibers and thus it cannot provide both benefits of the micro- and nano- scale fibers. Actually, nanofibers can mimic physically the extra cellular matrix (ECM). Cells are able to proliferate and spread well when seeded into nanofibers scaffold. However, cells face difficulty during migration in the nanofibers scaffold and this was the drawback of the nanofibers [8].

The benefit of the microfibers is that it is large enough to allow cells migration and this advantage is needed for the nanofibers scaffold. Therefore, in order to overcome the drawback of nanofibers, we need to add the microfibers into the nanofibers scaffold. The solution suggests to solution of the problem by fabricating multilayer scaffold or in other words microfibers/nanofibers layer by layer membranes through the multilayered electrospinning method [21].

According to Kumbar et al., fibroblast had higher proliferation rate on the scaffold which had diameter range between 350-1100nm [22]. In addition, Zhang et al. showed that the electrospun membranes which had fiber diameter ranging from $270 \mathrm{~nm}$ to $3250 \mathrm{~nm}$ were suitable for fibroblast to growth [23]. The average fiber diameter of $13 \% \quad \mathrm{w} / \mathrm{v}$ PCL and $8.8 \% \mathrm{w} / \mathrm{v}$ $\mathrm{PCL} /$ Chitosan layer by layer was $856.32 \mathrm{~nm}$ and was within the fiber diameter range which was suitable for the fibroblast to growth. The $13 \% \mathrm{w} / \mathrm{v}$ PCL and $8.8 \% \mathrm{w} / \mathrm{v}$ PCL/Chitosan layer by layer scaffold could have the potential to be used in skin tissue engineering.

Table 3 summarizes the water contact angle of microfibers for PCL, PCL/Chitosan as well as PCL and PCL/Chitosan. Pure PCL had a high

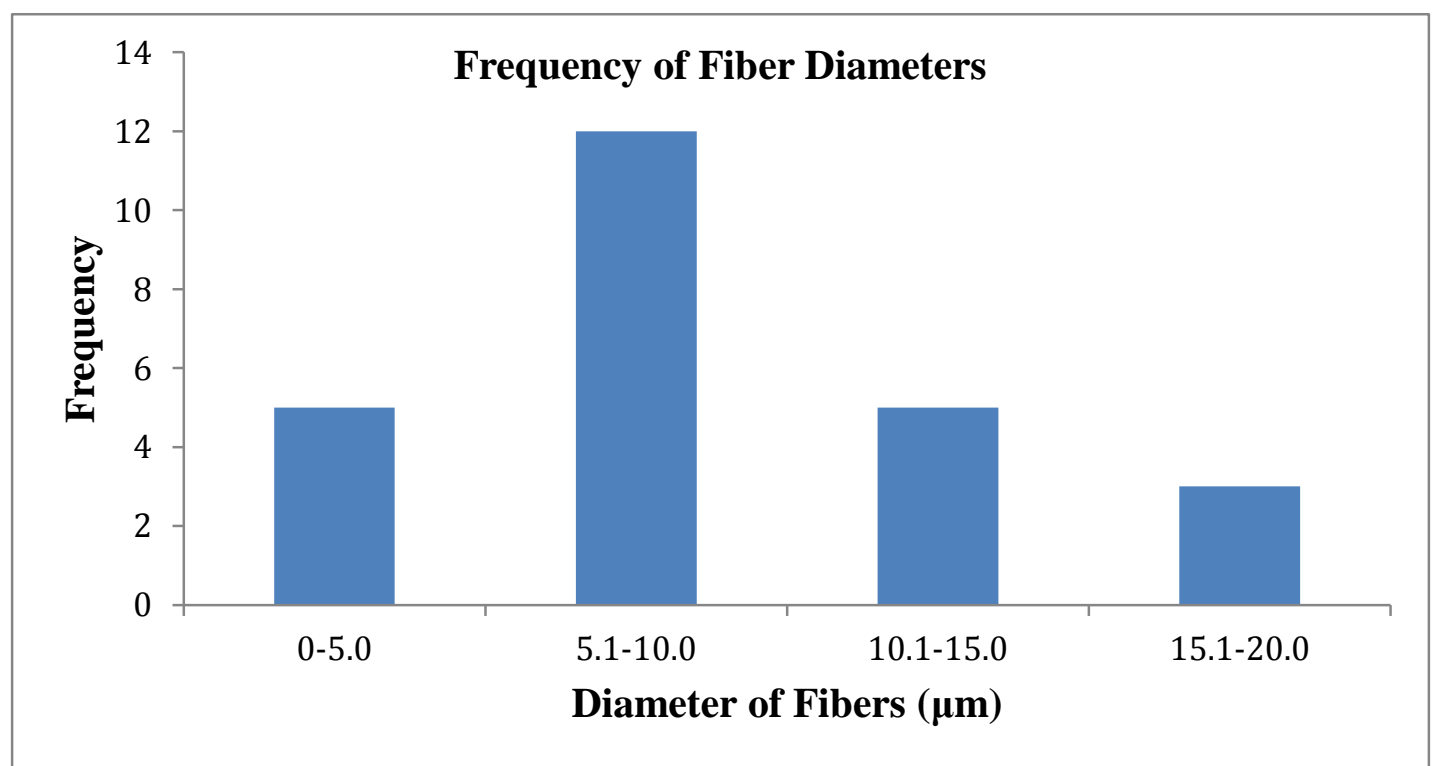

Figure 2 Distribution of Electrospun Fiber Diameters of 13\%w/v PCL and 8.8\%w/v PCL/Chitosan layer by layer membranes 
water contact angle which indicates the hydrophobic property of pure PCL membrane. Blending of chitosan with PCL decreased the water contact angle significantly in $\mathrm{PCL} /$ chitosan membrane. The double layered membrane had intermediate water contact angle than the other two membranes. Recent studies show that more hydrophilic surface of material will enhance cell adhesion on the surface [24, 25]. Therefore, good spreading, proliferation and differentiation of cells can be found on the hydrophilic surface [24]. The PCL and PCL/Chitosan layer by layer membrane had the improved wettability and thus it can enhance the cellular behavior when the membrane will be used in vitro or in vivo.

Figure 3 shows the percentages of water uptake by PCL, PCL /chitosan and the layer-by-layer membranes versus immersion time. It was observed that the amount of water uptake by the layered membrane was higher than the pure PCL membrane. The hydrophilicity of layer-by-layer membrane was higher than pure PCL electrospun membranes. According to Sultana et al., the amount of water uptake by the microfibrous membranes was depending on its hydrophilicity and the fluid taken in through the pores or capillaries of scaffolds [26]. PCL and PCL/Chitosan layer-by-layer was more hydrophilic than the pure PCL membrane due to the existence of the amino group present in chitosan and this chemical group interacted with surrounding water and formed hydrogen bond [27].

Table 3 The water contact angle of different membranes

\begin{tabular}{cc}
\hline Membranes & Contact angle $\mathbf{(}^{\mathbf{0}}$ ) \\
\hline $13.0 \% \mathrm{w} / \mathrm{v} \mathrm{PCL}$ & $130.45 \pm 4.04$ \\
$8.8 \% \mathrm{w} / \mathrm{v}$ & $59.57 \pm 3.32$ \\
$\mathrm{PCL} / \mathrm{Chitosan}$ & \\
& \\
& \\
13.0\%w/v PCL \\
and $8.8 \% \mathrm{w} / \mathrm{v}$ \\
$\mathrm{PCL} / \mathrm{Chitosan}$ \\
layer-by-layer
\end{tabular}




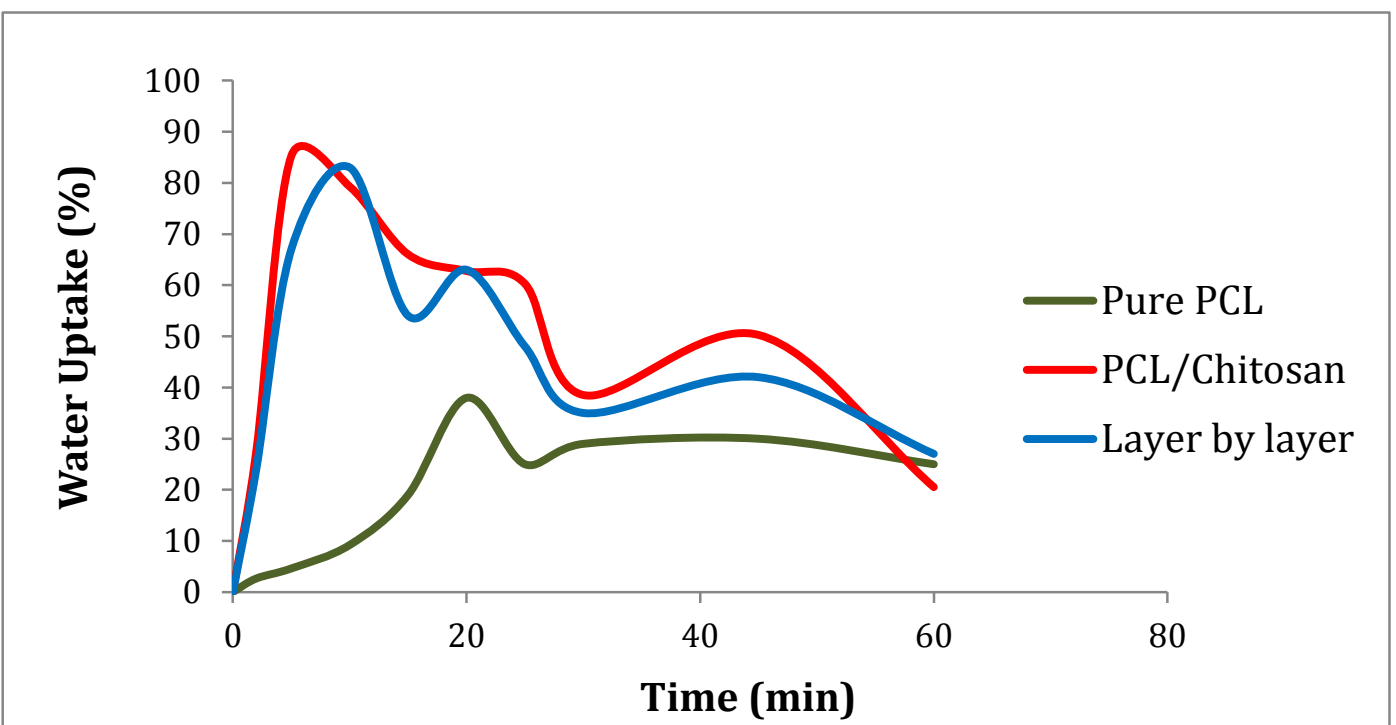

Figure 3 The Percentages of water uptake by Pure PCL, PCL/Chitosan and PCL and PCL/Chitosan membranes with immersion time

\subsection{CONCLUSION}

PCL and PCL/Chitosan micro/nano fibrous layer by layer electrospun membranes were successfully fabricated using multilayer electrospinning technique. The purpose of fabricating layer by layer electrospun membrane is to utilize both advantages of microfibers and nanofibers in single scaffold structure. The composite electrospun membranes had microfibers and nanofibers within single membrane. The average fiber diameter for the PCL and PCL/Chitosan micro/nano fibrous membranes doublelayered scaffold was $856.32 \mathrm{~nm}$ which may suitable for the growth of fibroblast. The water contact angle of the double layered composite electrospun membranes was lower than the pure PCL. It had higher water uptake properties compared to pure PCL membrane. The membranes could be potentially useful for skin tissue engineering application.

\section{ACKNOWLEDGEMENT}

The authors acknowledge Faculty Bioscience and Medical Engineering (FBME), Universiti Teknologi Malaysia (UTM), Skudai, Johor Bahru, Malaysia for the lab facilities. Authors also acknowledge GUP Tier 1 vot 06H35, FRGS vot 4F507, RMC, UTM, and Ministry of Education (MOE) for financial support.

\section{REFERENCES}

[1] Chen, A. A., V. L. Tsang, D. R. Albrecht, and S. N. Bhatia. 2007. 3-D fabrication technology for tissue engineering. In BioMEMS and Biomedical Nanotechnology. Springer. 23-38.

[2] Karp, J. M., P. D. Dalton, and M. S. Shoichet. 2003. Scaffolds for tissue engineering. MRS Bulletin. 28(04): 301-306.

[3] Leor, J., Y. Amsalem, and S. Cohen. 2005. Cells, scaffolds, and molecules for myocardial tissue engineering. Pharmacology \& therapeutics. 105(2): 151-163. 
[4] Nair, L. S. and C. T. Laurencin. 2007. Biodegradable Polymers as Biomaterials. Progress in Polymer Science. 32(8): 762798.

[5] Vroman, I. and L Tighzert. 2009. Biodegradable polymers. Materials. 2(2): 307-344.

[6] Gomes, M. E., H. L. Holtorf, R. L. Ries, and A. G. Mikos. 2006. Influence of the porosity of starch-based fiber mesh scaffolds on the proliferation and osteogenic differentiation of bone marrow stromal cells cultured in a flow perfusion bioreactor. Tissue Engineering. 12(4): 801-809.

[7] Tuzlakoglu, K., N. Bolgen, A. J. Salgodam, M. E. Gomes, E. Piskin, and R. L. Reis. 2005. Nano-and micro-fiber combined scaffolds: a new architecture for bone tissue engineering. Journal of Materials Science: Materials in Medicine. 16(12): 1099-1104.

[8] Mota, C., D. Puppi, D. Dinucci, C. Errico, P. Bartolo, and F. Chiellini. 2011. Dual-scale polymeric constructs as scaffolds for tissue engineering. Materials. 4(3): 527-542.

[9] Puppi, D., A. M. Piras, F. Chiellini, E. Chieillini, A. Martin, I. B. Leonor, N. Neves, and R. Peis. 2011. Optimized electroand wet-spinning techniques for the production of polymeric fibrous scaffolds loaded with bisphosphonate and hydroxyapatite. Journal of Tissue Engineering and Regenerative Medicine. 5(4): 253-263.

[10] Middleton, J. C. and A. J. Tipton. 2000. Synthetic Biodegradable Polymers as Orthopedic Devices. Biomaterials. 21(23): 23352346.

[11] Gunatillake, P. A. and Adhikari, R. 2003. Biodegradable synthetic polymers for tissue engineering. Eur Cell Mater. 5(1): 1-16.

[12] Sarasam, A. and Madihally, S. V. 2005. Characterization of chitosan-polycaprolactone blends for tissue engineering applications. Biomaterials. 26(27): 5500-5508.

[13] Suyatma, N. E., A. Copinet, L. Tighzert, and V. Coma. 2004. Mechanical and barrier properties of biodegradable films made from chitosan and poly (lactic acid) blends. Journal of Polymers and the Environment. 12(1): 1-6.

[14] Deng, D., P. Jiao, X. Ye, and L. Xia. 2012. An image-based model of the whole human heart with detailed anatomical structure and fiber orientation. Computational and Mathematical Methods in Medicine.

[15] Butler, B., P. J. Vergano, R. F. Testin, J. M. Bunn, and J. L. Wiles. 1996. Mechanical and barrier properties of edible chitosan films as affected by composition and storage. Journal of Food Science. 61(5): 953-956.

[16] Hosokawa, J., M. Nishiyama, K. Yoshihara, and T. Kubo. 1990. Biodegradable film derived from chitosan and homogenized cellulose. Industrial \& Engineering Chemistry Research. 29(5): 800-805.

[17] Chen, G., T. Ushida, and T. Tateishi. 2002. Scaffold design for tissue engineering. Macromolecular Bioscience. 2(2): 67-77.

[18] Borm, P. J., D. Robbins, S. Houbold, T. Kuhlbusch, H. Fissan, K. Donaldson, R. Schins, V. Stones, W. Kreyling, and J. Lademann. 2006. The potential risks of nanomaterials. Particle and Fibre Toxicology. 3(1): 11.

[19] Wang, Z.M. 2008. Onedimensional nanostructures. Vol. 
3. Springer Science \& Business Media.

[20] Chen, H., A. G. Mikos, Q. P. Pham, U. Sharma, and Z. P. Luo. Finite Element Analyses of Flow Field in Multilayer Nanofiber/Microfiber Scaffolds.

[21] Pham, Q. P., U. Sharma, and A. G. Mikos. 2006. Electrospun poly (E-caprolactone) microfiber and multilayer nanofiber/microfiber scaffolds: characterization of scaffolds and measurement of cellular infiltration. Biomacromolecules. $\quad 7(10)$ : 2796-2805.

[22] Kumbar, S. G., S. P. Nukavarapu, R. James, L. S. Nair, and C. T. Laurancin. 2008. Electrospun poly (lactic acid-co-glycolic acid) scaffolds for skin tissue engineering. Biomaterials. 29(30): 4100-4107.

[23] Zhang, B. 2011. Electrospun Poly (2-Hydroxyethyl Methacrylate) Nanofibrous Scaffolds for Skin Tissue Engineering. The University of Akron.

[24] Travan, A., E. Marsich, I. Donati, M. P. Foulc, N. Moritz, H. T. Aro,
S. Paoletti. 2012. Polysaccharidecoated thermosets for orthopedic applications: from material characterization to in vivo tests. Biomacromolecules. $13(5)$ : 1564-1572

[25] Goddard, J. M. and J. Hotchkiss. 2007. Polymer surface modification for the attachment of bioactive compounds. Progress in Polymer Science. 32(7): 698-725.

[26] Sultana, N. and T. H. Khan. 2013. Water absorption and diffusion characteristics of nanohydroxyapatite (nHA) and poly (hydroxybutyrate-cohydroxyvalerate-) based composite tissue engineering scaffolds and nonporous thin films. Journal of Nanomaterials. 2013: 1-8.

[27] Mad Jin, R., N. Sultana, S. Baba, S. Hamdan, and A.F. Ismail. 2015. Porous PCL/Chitosan and nHA/PCL/Chitosan Scaffolds for Tissue Engineering Applications: Fabrication and Evaluation. Journal of Nanomaterials. 2015: $1-8$. 